\title{
Extracorporeal Shock Wave Therapy in Dupuytren's Disease
}

\author{
Serkan Aykut, ${ }^{1}$ Canan Aydın, ${ }^{2}$ Kahraman Öztürk, ${ }^{1}$ Fatih Arslanoğlu, ${ }^{3}$ Cem Yalın Kılınç \\ 'Department of Hand and Upper Extremity Surgery, Health Sciences University, Metin Sabanci Baltalimani Bone Diseases Training and \\ Research Hospital, Istanbul, Turkey \\ ${ }^{2}$ Department of Sports Medicine, Health Sciences University, Metin Sabanci Baltalimani Bone Diseases Training and Research Hospital, \\ Istanbul, Turkey \\ ${ }^{3}$ Department of Orthopedics and Traumatology, Selahaddin Eyyubi Public Hospital, Istanbul, Turkey \\ ${ }^{4}$ Department of Orthopedics and Traumatology, Sitki Kocman University, Training and Research Hospital, Istanbul, Turkey
}

\begin{abstract}
Objectives: We investigated the use of extracorporeal shock wave therapy (ESWT) in patients with Dupuytren's Disease (DD) palmar nodules in an attempt to reduce the contracture, alleviate the pain (if any), increase the range of motion and quality of life, and delay a probable surgery in the long term.

Methods: Patients with DD who presented to our hand surgery clinic and fulfilled the inclusion criteria were enrolled. The treatment was performed by the same physician using the ESWT device once a week for six weeks. The patients were evaluated with the VAS score, Quick-DASH questionnaire, and MAYO wrist score, and their grip strength was measured using a Jamar dynamometer.

Results: The mean age of the 23 patients included in the study was 51 years. There was a significant improvement in the second measurement of VAS and DASH scores compared with the preoperative values. The increase in the second and final follow-up measurements of the MAYO score and grip strength results compared with the preoperative values was found significant. The table-top test results turned negative in 16 patients.

Conclusion: We can suggest that ESWT in the early term can be preferred over costly injections and surgical intervention options as it increases the quality of life and delays the recurrence of contractures.

Keywords: Dupuytren's disease; extracorporeal shockwave therapy; treatment.

Please cite this article as "Aykut S., Aydın C., Özturk K., Arslanoğlu F., Kılınç C.Y. Extracorporeal Shock Wave Therapy in Dupuytren's Disease. Med Bull Sisli Etfal Hosp 2018;52(2):124-128".
\end{abstract}

$\mathrm{D}$ upuytren's disease (DD), also known as palmar fibromatosis, is a proliferative fibroplasia of palmar aponeurosis. The condition is a major cause of morbidity and causes progressive and irreversible flexion contractures in the fingers, which in turn affects patients' daily activities and reduces their quality of life. Although the etiology of DD is unknown, it has been shown to originate from an autosomal dominant inheritance with incomplete penetrance. ${ }^{[1]}$
Diabetes mellitus, long-term use of anticonvulsants due to epilepsy, liver disease, HIV infection, complex regional pain syndrome, myocardial infarction, alcohol consumption and/or smoking, and trauma are the risk factors for DD. The disease is clinically categorized into early, active, and advanced stages. In the early stage, the integrity of the skin is compromised. Nodules and cords are seen during the active stage. In the advanced stage, fibrocytes and contracture develop. ${ }^{[2-4]}$ 
As the treatment modalities for the disease are still a matter of debate, medical therapy and physiotherapy modalities are preferred in the early stage, while collagenase injection and radiotherapy, dimethyl sulfoxide, topical use of Vitamin $A$ and $E$, hyperbaric oxygen application, corticosteroids, gamma-interferon, and 5-fluorouracil applications are applied in the advanced stages. Surgical options are considered for joint contractures, which may affect daily activities. $^{[5,6]}$

Despite the success of surgical interventions, alternative medical treatment methods have been investigated, as the condition has a high recurrence rate after surgery. One of these alternative methods is the extracorporeal shock wave therapy (ESWT). We planned our current study taking another study from 2011 as a reference, whose hypothesis was based on the use of ESWT in DD. ${ }^{[7]}$ In the current study, we investigated the use of ESWT in patients with palmar nodules in an attempt to reduce the contracture, alleviate the pain (if any), increase the range of motion and quality of life, and delay a probable surgery in the long term.

\section{Methods}

This study was approved by our hospital's ethical committee. All patients signed a free and informed consent form. Patients with DD who presented to our hand surgery clinic and fulfilled the following inclusion criteria were enrolled:

- Diagnosis of DD in a minimum of one finger

- Patients who were able to attend the follow-up visits

- Patients aged $\geq 18$ years

- Those with a flexion of $\leq 30^{\circ}$ in the metacarpophalangeal (MP) joint and without a contracture in the proximal interphalangeal (PIP) joint

The exclusion criteria were as follows:

- Patients who were pregnant or planned of getting pregnant during the treatment period

- Presence of a chronic, neurological, or neuromuscular disorder that affected the hands

- Patients who did not accept to receive a treatment for their contractures

- $\quad$ Patients who were contraindicated for ESWT

Loss of extension in the involved MP, PIP, Distal interphalangeal (DIP) joints due to the contracture cords were evaluated in the examinations of the patients. All patients underwent the table-top test. The ESWT application was performed with the patient sitting on a gurney with the elbow flexed at $30^{\circ}$, the forearm in supination, and the probe of the ESWT device positioned $90^{\circ}$ tangent to the pain site. (Fig. 1) The physician who performed the ESWT and the pa- tient had to wear earmuffs as the device operated with high noise. The application site was cleaned with povidone-iodine solution, and a gel was applied on the skin to enhance conductivity. None of the patients were administered local anesthesia. The regions with nodules were marked, and the probe was circularly moved around this mark. The treatment was performed by the same physician using the ESWT device (Roland E-SWT 2; Elettronica Pagani srl, Milan, Italy) once a week for six weeks, run at a frequency of 6-8 $\mathrm{Hz}$ with 1700 pulse/session at $0.18 \mathrm{mj} / \mathrm{cm}^{2}$. No hot or cold therapy was performed following ESWT application. None of the patients encountered the rarely seen symptoms of redness, pain, or swelling.

The patients were evaluated with the VAS score, QuickDASH questionnaire, and MAYO wrist score. Their grip strength was measured thrice using a Jamar dynamometer (Sammons Preston, Inc., Bolingbrook, IL, USA) with one minute intervals between measurements, and the average of the three measurements was noted.

The mean, standard deviation, median, minimum, and maximum values were considered in the descriptive statistical analysis of our data. Analysis of the repeating measurements was performed with the Wilcoxon test. Analyses were performed using the SPSS 22.0 software.

\section{Results}

The mean age of the 23 (17 male, six female) patients included in the study was 51 (range: 20-74) years. The five patients who had bilateral involvements were all males. Fourteen cases had contracture in the fourth finger, nine had in the third finger, and five had in the fifth finger.

There was a significant improvement in the second mea-

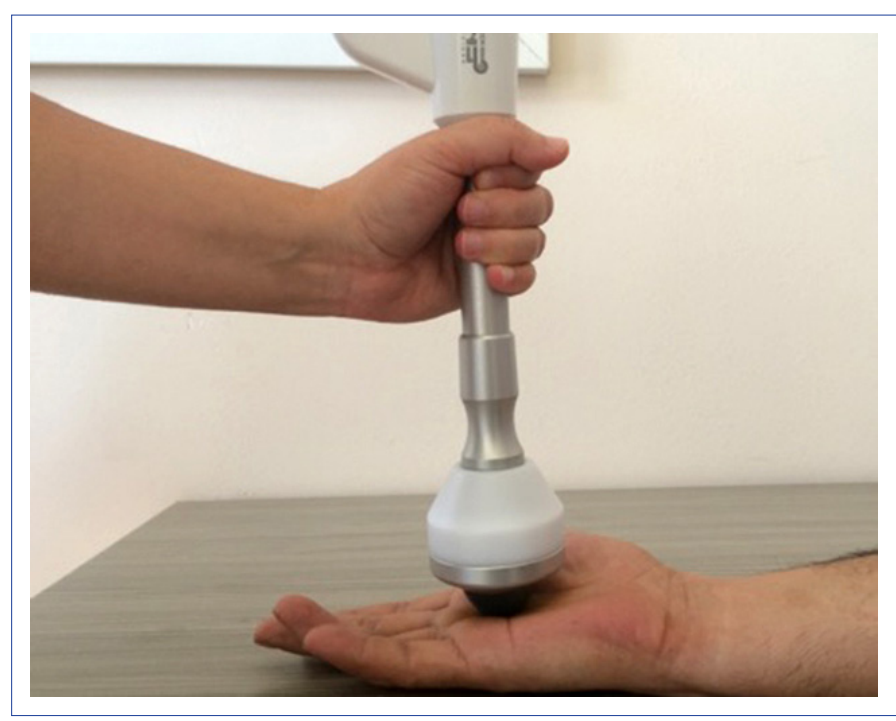

Figure 1. Extracorporeal shock wave therapy (ESWT) application. 
Table 1. Wilcoxon test $p<0.05$

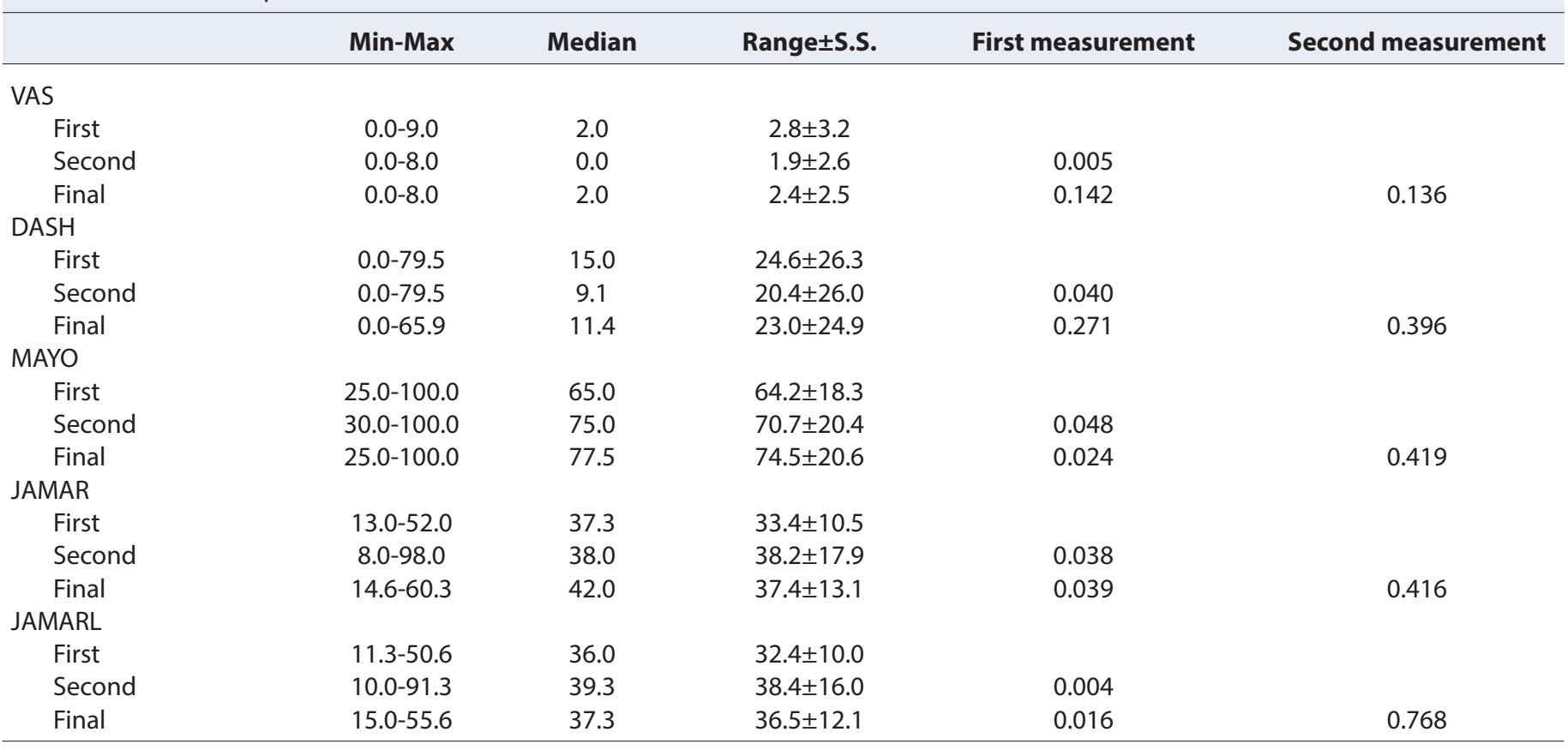

surement of VAS and DASH scores in comparison to the preoperative values $(p<0.05)$; however, no statistically significant difference was detected at the final follow-up measurements (Table 1, Fig. 2). Conversely, the increase in

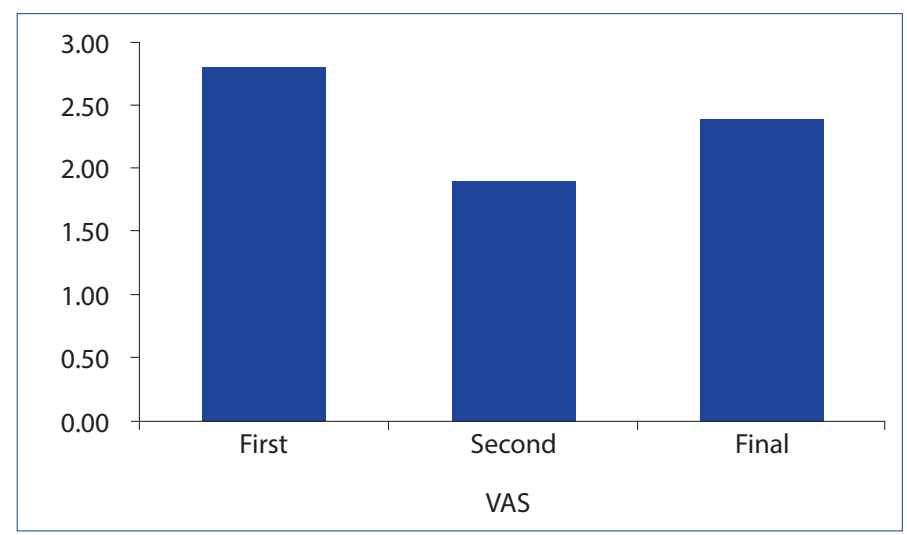

Figure 2. First and follow-up VAS results.

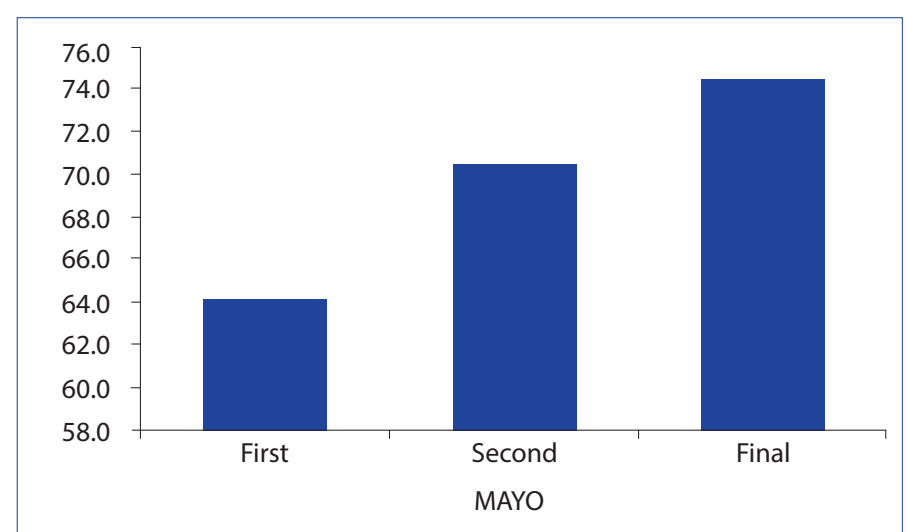

Figure 3. First and follow-up MAYO results. the second and final follow-up measurements of the MAYO score and grip strength results in comparison to the preoperative values was found significant. However, no significant difference was observed between the second and final follow-up measurements (Figs. 3, 4). The table-top test results turned negative in 16 patients.

\section{Discussion}

Regarding management of DD, which still lacks a universal treatment protocol and is usually managed with injections and surgical interventions in the advanced stage, we achieved a significant functional recovery in the early term using ESWT. In accordance with the literature, the prevalence of DD was higher in our male population and peaked at the fifth decade. It has been reported that the Dupuytren's contracture mostly involves the fourth finger. ${ }^{[8,9]}$ In

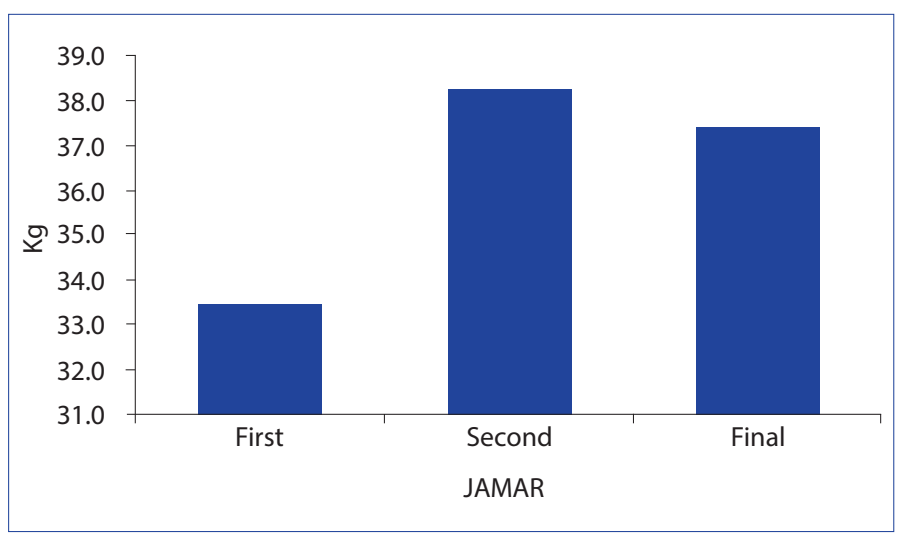

Figure 4. First and follow-up grip strength results. 
our study, the fourth finger was either involved alone or together with other fingers in 14 patients. Six of our patients had a positive family history.

The gold standard in the surgical management of DD is the excision of the affected tissue and the correction of finger contractures, if any. ${ }^{[10]}$ In a systematic review by Werker et al., it was shown that the rate of success in correction of the contractures varied between $15 \%$ and $96 \%$, while the rate of recurrence was found between $12 \%$ and $100 \%$, following surgeries performed at different stages of the disease. ${ }^{[11]}$ In another systematic review by Chen et al., ${ }^{[12]}$ the rate of recurrence was $12 \%-39 \%$ in a follow-up period of 1.5-7.3 years following open partial fasciectomy, $50 \%-58 \%$ in a follow-up period of 3-5 years following needle aponeurotomy, and $10 \%-31 \%$ in a follow-up period of 3 months to 4 years following collagenase clostridium histolyticum $(\mathrm{CCH})$ treatment.

Treatment with CCH (Xiaflex) has been clinically approved by the US Food and Drug Administration. Collagenase injections are also called enzymatic fasciotomy. After 8 years of follow-up of eight enzymatic fasciotomy patients, Watt et al. found that the contracture had increased by a mean of $23^{\circ}$ in the MP joint $(n=6)$ and $60^{\circ}$ in the PIP joint $(n=2) .^{[13]}$ Although the authors had observed recurrences throughout their follow-up, they underlined the fact that these were less severe contractures than the original ones. In another study, McCarthy et al. concluded that enzymatic fasciotomy was not superior to surgical fasciotomy. ${ }^{[14]}$

There is no study in the literature investigating the use of ESWT in the management of DD. When we look into the use of ESWT in other conditions, we see that Knobloch et al. ${ }^{[7]}$ The authors also hypothesized that the technique could be applied in a similar fibromatous disease such as DD. The literature holds no validated questionnaire designed for DD. The DASH questionnaire, being a subscale of the SF-36, has a reasonable validity. We used this questionnaire in our study. ${ }^{[15]}$ In an animal model for Peyronie's disease, 2000 shockwaves were applied. ${ }^{[16]}$ Using the technique in 44 patients with Peyronie's disease in their controlled study, they achieved a reduction in pain, significant recovery in erectile function, and an increase in quality of life. ${ }^{[17]}$ The same study clinical randomized controlled trial in Peyronie's disease shock waves significantly reduced pain and improved erectile function and quality of life. ${ }^{[18]}$

In another study of theirs, Knobloch et al. ${ }^{[19]}$ reported reduction of pain and softening of the nodules in the third month following the application of ESWT in six patients with painful plantar fibromatosis (Ledderhose's disease).

The limitations of our study are its short follow-up period and small number of patients. However, by sharing our sig- nificant early-term results, we wanted to pave the way for future studies with larger series and longer follow-up periods. When similar fibroproliferative diseases are considered, it can be contemplated that ESWT can be used in Peyronie's disease, Garrod's pads, and Ledderhose's disease.

\section{Conclusion}

A definite treatment for DD still does not exist. Corrective surgeries may loosen the contractures, lessen the symptoms, and increase the quality of life. Recurrence is a common and inevitable complication of all treatment modalities available. We can suggest that ESWT in the early term can be preferred over costly injections and surgical intervention options as it increases the quality of life and delays the recurrence of contractures. We believe a valid treatment protocol for ESWT should be established with further randomized controlled series with long-term outcomes.

\section{Disclosures}

Ethics Committee Approval: The study was approved by the Local Ethics Committee.

Peer-review: Externally peer-reviewed.

Conflict of Interest: None declared.

Authorship contributions: Concept - S.A., C.A.; Design - S.A., C.A.; Supervision - S.A., C.A., K.Ö.; Materials - S.A., C.A., F.A.; Data collection \&/or processing - S.A., C.A., F.A., C.Y.K.; Analysis and/or interpretation - S.A., C.A.; Literature search - S.A., C.A., F.A., C.Y.K.; Writing - S.A., C.A.; Critical review - S.A., C.A., K.Ö.

\section{References}

1. Hu FZ, Nystrom A, Ahmed A, Palmquist M, Dopico R, Mossberg I, et al. Mapping of an autosomal dominant gene for Dupuytren's contracture to chromosome $16 \mathrm{q}$ in a Swedish family. Clin Genet 2005;68:424-9.

2. McGrouther DA. Dupuytren's contracture. In: Green DP, Hotchkiss RN, Pederson WC, editors. Operative Hand Surgery. 4th ed. New York: Churchill Livingstone; 1999. p. 563-91.

3. Umlas ME, Bischoff RJ, Gelberman RH. Predictors of neurovascular displacement in hands with Dupuytren's contracture. J Hand Surg Br 1994;19:664-6.

4. Short WH, Watson HK. Prediction of the spiral nerve in Dupuytren's contracture. J Hand Surg Am 1982;7:84-6.

5. Bansal V, Naidu SH. Dupuytren's disease. Curr Opin Orthop 2005;16:236-9.

6. Badalamente MA, Hurst LC, Hentz VR. Collagen as a clinical target: nonoperative treatment of Dupuytren's disease. J Hand Surg Am 2002;27:788-98.

7. Knobloch K, Kuehn M, Vogt PM. Focused extracorporeal shockwave therapy in Dupuytren's disease--a hypothesis. Med Hypotheses 2011;76:635-7.

8. Dominguez-Malagon HR, Alfeiran-Ruiz A, Chavarria-Xicotencatl P, 
Duran-Hernandez MS. Clinical and cellular effects of colchicine in fibromatosis. Cancer 1992;69:2478-83.

9. Luck JV. Dupuytren's contracture; a new concept of the pathogenesis correlated with surgical management. J Bone Joint Surg Am 1959;41-A:635-64.

10. Badalamente MA, Hurst LC. Enzyme injection as nonsurgical treatment of Dupuytren's disease. J Hand Surg Am 2000;25:629-36.

11. Werker PM, Pess GM, van Rijssen AL, Denkler K. Correction of contracture and recurrence rates of Dupuytren contracture following invasive treatment: the importance of clear definitions. J Hand Surg Am 2012;37:2095-105.

12. Chen NC, Srinivasan RC, Shauver MJ, Chung KC. A systematic review of outcomes of fasciotomy, aponeurotomy, and collagenase treatments for Dupuytren's contracture. Hand (NY) 2011;6:250-5.

13. Watt AJ, Curtin CM, Hentz VR. Collagenase injection as nonsurgical treatment of Dupuytren's disease: 8-year follow-up. J Hand Surg Am 2010;35:534-9, 539.

14. McCarthy DM. The long-term results of enzymic fasciotomy. J
Hand Surg Br 1992; 178:356.

15. SooHoo NF, McDonald AP, Seiler JG 3rd, McGillivary GR. Evaluation of the construct validity of the DASH questionnaire by correlation to the SF-36. J Hand Surg Am 2002;27:537-41.

16. Andrade E, Cortez I, Claro J, Pompeu E, Leite K, Paranhos M, et al. Preliminary findings from a new animal model for Peyronie's disease involving extracorporeal shock waves. BJU Int 2009;103:1104-6.

17. Srirangam SJ, Manikandan R, Hussain J, Collins GN, O'Reilly PH. Long-term results of extracorporeal shockwave therapy for Peyronie's disease. J Endourol 2006;20:880-4.

18. Palmieri A, Imbimbo C, Longo N, Fusco F, Verze P, Mangiapia F, et al. A first prospective, randomized, double-blind, placebo-controlled clinical trial evaluating extracorporeal shock wave therapy for the treatment of Peyronie's disease. Eur Urol 2009;56:363-9.

19. Knobloch K, Vogt PM. High-energy focussed extracorporeal shockwave therapy reduces pain in plantar fibromatosis (Ledderhose's disease). BMC Res Notes 2012;5:542. 